\title{
EVALUATION OF PATHOLOGICAL COMPLETE RESPONSE IN PATIENTS WITH BREAST CANCER SUBMITTED TO NEOADJUVANT CHEMOTHERAPY AND SURGERY IN HOSPITAL DO CÂNCER DO MATO GROSSO
}

Clarissa Resende Correa', Luis Fernando Correa Barros², Sheila Queiroz Campos²

'Universidade Federal do Rio Grande - Rio Grande (RS), Brazil.

${ }^{2}$ Hospital do Cancer do Mato Grosso - Cuiabá (MT), Brazil.

This study aims at comparing the pathological complete response rate of women submitted to neoadjuvant chemotherapy for breast cancer in the world with the community from Hospital do Câncer do Mato Grosso, to verify which histological subtypes have higher chances of reaching the pathological complete response. Therefore, we will analyze the medical records of the patients who underwent neoadjuvant chemotherapy followed by surgery. Besides the initial clinical staging from I to III between 2014 to 209. The criteria in RECIST 1.1 were used (Response Evaluation Criteria in Solid Tumors) to define pathological complete response for the breast and the axilla. We concluded that the benefit of neoadjuvant chemotherapy is mostly seen in patients with negative hormone receptors and positive HER2, and they have higher chances of reaching pathological complete response. In the triple negative subtype, neoadjuvant chemotherapy with the objective of reaching $\mathrm{pCR}$ is, until this moment, the best strategy for higher survival rates in these patients, according to data from the global literature. 